\title{
Progress on Gastrointestinal Symptoms, Treatment and Protection in COVID-19 Patients
}

\author{
Tian Huang $1,2,3 \#$, Long-Quan Li ${ }^{1,2,3 \#}$, Yong-Qing Wang ${ }^{4 \#}$, Zheng-Ping Wang ${ }^{5}$, \\ Yuan Liang ${ }^{1,2,3}$, Tao-Bi Huang ${ }^{1,2,3}$, Hui-Yun Zhang ${ }^{1,2,3}$, \\ Wei-Ming Sun ${ }^{* *}$ and Yu-Ping Wang ${ }^{2,3 *}$
}

\begin{abstract}
${ }^{1}$ The First Clinical Medical School, Lanzhou University, Lanzhou, China; ${ }^{2}$ Department of Gastroenterology, The First Hospital of Lanzhou University, Lanzhou, China; ${ }^{3}$ Key Laboratory for Gastrointestinal Diseases, Gansu Province, The First Hospital of Lanzhou University, Lanzhou, China; ${ }^{4}$ Day Diagnostic Center, Gansu Provincial Hospital, Lanzhou, China; ${ }^{5}$ Clinical College of Chinese Medicine, Gansu

University of Chinese Medicine, Lanzhou, China; ${ }^{6}$ Department of Endocrinology, The First Hospital of Lanzhou University, Lanzhou, China; "These authors contributed equally to this work.
\end{abstract}

\begin{abstract}
Coronavirus disease 2019 (COVID-19) caused by the severe acute respiratory syndrome coronavirus-2 (SARSCoV-2) induced a worldwide pandemic. The main clinical manifestations of COVID-19 patients have been fever, cough, dyspnea, and other respiratory symptoms. However, some patients' initial symptoms have been nausea, vomiting, diarrhea and other gastrointestinal symptoms, and SARS-CoV-2 RNA could be found in their stool samples. Studies have shown that the gastrointestinal tract highly-expressed angiotensin-converting enzyme 2 is used by SARS-CoV-2 to enter cells. Therefore, exploring the damage caused by SARS-CoV-2 to the gastrointestinal tract and whether it could replicate in the gastrointestinal tract and transmit through fecal-oral route has significance for the diagnosis, treatment and prevention of COVID-19. We combined the current clinical data about COVID-19 patients with gastrointestinal symptoms as well as its pathogenic mechanism and prevention methods herein to review the relationship between the disease and gastrointestinal symptoms.
\end{abstract}

Introduction

As of 20 April 2020, the severe acute respiratory syndrome coronavirus-2 (SARS-CoV-2) has infected more than 22,256,220 people worldwide and killed more than 782,456 . The disease caused by SARS-CoV-2 was named coronavirus disease 2019 (COVID-19) by the World Health Organization. ${ }^{1}$ Scientists have divided the coronaviruses into four groups, namely alpha, beta, gamma, and delta. ${ }^{2}$ SARS-CoV-2 and Middle East respiratory syndrome coronavirus (MERS-CoV) are beta-coronaviruses. SARS-CoV-2 spreads the

Keywords: SARS-CoV-2; COVID-19; Gastrointestinal symptoms; Diarrhea, Fecaloral transmission.

Abbreviations: COVID-19, coronavirus disease 2019; SARS-CoV-2, severe acute respiratory syndrome coronavirus-2; ACE2, angiotensin-converting enzyme 2; ACEI, angiotensin converting enzyme inhibitor; ARB, angiotensin receptor blocker.

Received: July 22, 2020; Revised: August 26, 2020; Accepted: September 11, 2020

${ }^{*}$ Correspondence to: Yu-Ping Wang, Department of Gastroenterology, The First Hospital of Lanzhou University; Key Laboratory for Gastrointestinal Diseases, Gansu Province, The First Hospital of Lanzhou University, No. 1 West Donggang Road, Lanzhou, Gansu 730000, China. E-mail: wangyuping@1zu.edu.cn; Wei-Ming Sun, Department of Endocrinology, The First Hospital of Lanzhou University, No. 1 West Donggang Road, Lanzhou, Gansu 730000, China. E-mail: swm77@163.com

How to cite this article: Huang T, Li L-Q, Wang Y-Q, Wang Z-P, Liang Y, Huang T-B, Zhang H-Y, Sun W-M, Wang Y-P. Progress on Gastrointestinal Symptoms, Treatment and Protection in COVID-19 Patients. Exploratory Research and Hypothesis in Medicine 2020;5(4):134-138. doi: 10.14218/ERHM.2020.00049. fastest among them, and its reproduction number (R0) was estimated at $5.7,{ }^{3}$ which meant that with no external intervention and immunity, an average of 5.71 humans would become infected by SARS-CoV-2. The up-to-date guideline noted the infection source of COVID-19 to be mainly patients infected by SARS-CoV-2 and having asymptomatic infections; in addition to the common respiratory droplets and close contact transmission routes, we should pay attention to the fact that SARS-CoV-2 can be detected in feces and urine, and environmental pollution caused by human excrement can result in the spread of this virus and disease. ${ }^{4}$ The World Health Organization proclaimed that COVID-19 was a Public Health Emergency of International Concern, ${ }^{\mathbf{5}}$ and it became recognized as a major threat to public health around the world.

SARS-CoV-2 infects organs by combining with the angiotensin-converting enzyme 2 (ACE2) receptor on a cell expressing its ligand. SARS-CoV-2 has the same cell entry receptor ACE2 as SARS-CoV. ${ }^{6} \mathrm{Li}$ et al. $^{7}$ analyzed the expression of ACE2 using datasets from the Genotype-Tissue Expression (GTEx) project and showed that ACE2 was expressed highest in the small intestine, testis, kidneys and heart, lowest in the blood, spleen, bone marrow, brain, blood vessels and muscle, and medium in the lungs, colon, liver and bladder (Fig. 1). Thus, COVID-19 patients' clinical manifestations can vary from asymptomatic to multiorgan and systemic dysfunctions. ${ }^{8}$ The overall damage mechanism can be summarized as direct damage caused by the virus and a variety of indirect damage mechanisms, including immune-mediated damage, hypoxia, in- 


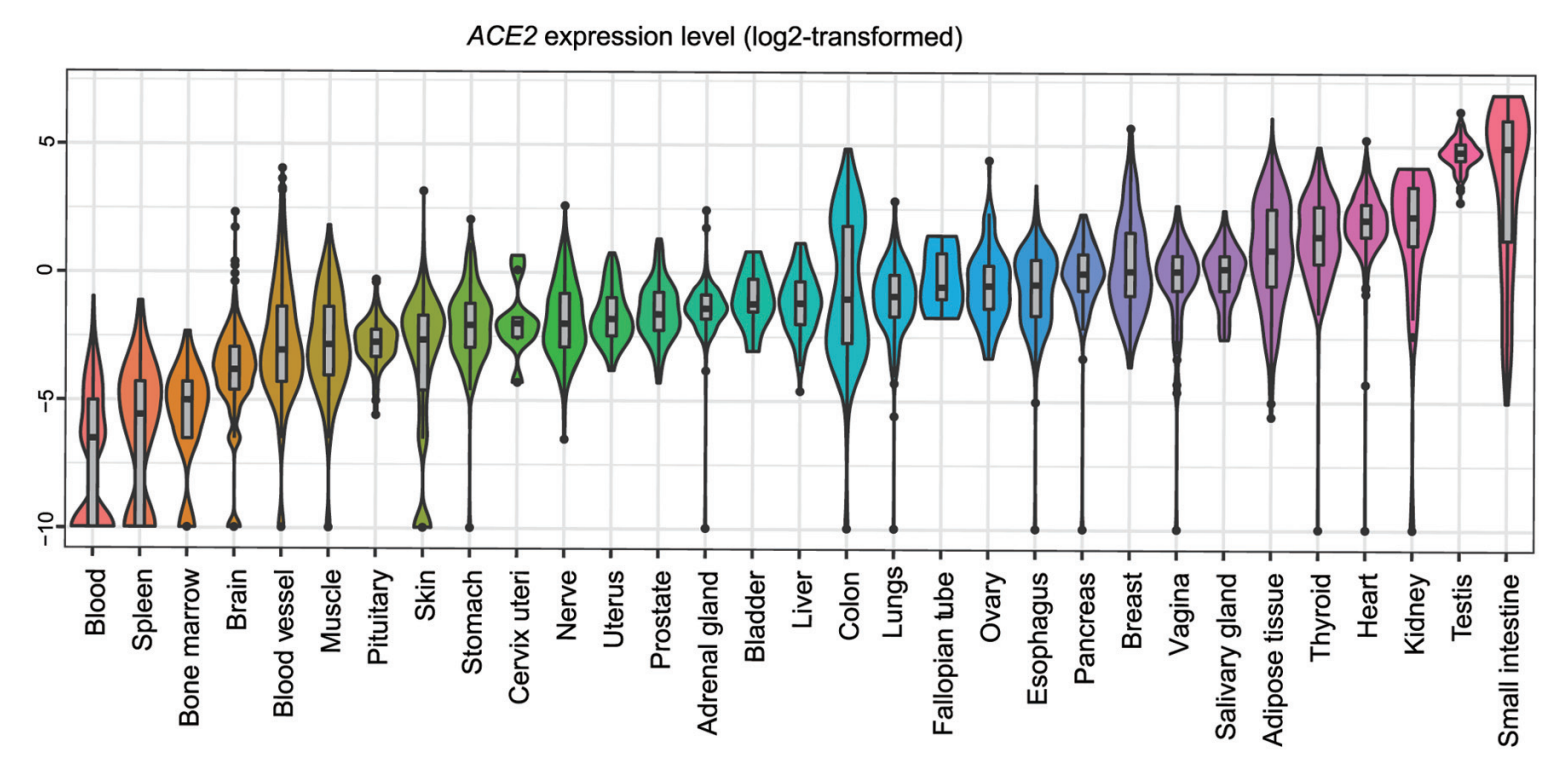

Fig. 1. ACE-2 expression level in different organs. ${ }^{7}$ (The authors have obtained the permision of re-use this figure).

flammatory factor storm, abnormal blood coagulation, and so on. ${ }^{9-11}$

The main clinical manifestations of COVID-19 patients have been fever, cough, dyspnea, and other respiratory symptoms. However, a study pointed out many patients presenting with nausea, vomiting, diarrhea and other gastrointestinal symptoms as the initial symptoms. ${ }^{12}$ Viral protein has been detected in the gastrointestinal epithelium, ${ }^{13}$ and SARS-CoV-2 RNA has been detected in stool samples of infected patients. ${ }^{14,15}$ These results suggested gastrointestinal cells as potential targets of SARS-CoV-2 and the potential for virus replication in such.

Although SARS-CoV-2 can cause damage to multiple organs, given the existence of a majority of patients with atypical gastrointestinal symptoms and the possibility of a fecal-oral transmission route, our study reviewed the clinical manifestations in the gastrointestinal tract, mechanisms and treatment of gastrointestinal tract injury, and protection measures based on the studies of COVID-19 to date.

\section{Gastrointestinal symptoms}

Gastrointestinal symptoms might be the single manifestation of COVID-19 patients, and a majority of patients infected by SARSCoV-2 have been admitted to the gastroenterology department. ${ }^{16}$ If physicians do not pay enough attention to these patients, it might lead to delayed diagnosis and inadequate management of infected patients. About $10 \%$ of patients develop diarrhea and nausea 1-2 days before the onset of fever and respiratory symptoms. ${ }^{17} \mathrm{~A}$ study by Wang et al. ${ }^{17}$ supported the possibility that diarrhea might be an earlier clinical symptom of the COVID-19 patient, possibly prior to fever or respiratory symptoms. What is more, with the progression of the disease, the gastrointestinal symptoms' occurrence rate also increases. ${ }^{18}$ The definition of diarrhea was 3 or more loose/liquid stools per day or an increase in the number of evacuations compared with the usual. ${ }^{19}$ The incidence rate of gastrointestinal symptoms in COVID-19 patients has varied in different studies, which reflects that clinical workers paid different degree of attention to COVID-19 patients' gastrointestinal symptoms. A study including 140 COVID-19 patients in Wuhan showed that gastrointestinal symptoms were found in up to $39.6 \%$ of these patients, nausea in $24(17.3 \%)$, diarrhea in $18(12.9 \%)$, and vomiting in $7(5.0 \%) .{ }^{20}$ Among 74 COVID-19 patients with gastrointestinal symptoms in the Zhejiang province, $53(71.6 \%)$ had the symptom of diarrhea only, 11 (14.9\%) only had vomiting and $10(13.5 \%)$ only had nausea. ${ }^{21}$ Our previous meta-analysis collected 10 articles and included 1994 COVID-19 patients, and showed that $95(4.8 \%)$ and $78(3.9 \%)$ patients had diarrhea or nausea and vomiting, respectively. ${ }^{22}$ A study that collected data of 1,099 patients reported nausea or vomiting in 55 $(5.0 \%)$ and diarrhea in $42(3.8 \%) .{ }^{23} \mathrm{~A}$ meta-analysis comprising 13,251 patients showed that the most common gastrointestinal symptoms were anorexia (10.2\%), diarrhea $(8.4 \%)$, and nausea $(5.7 \%) .{ }^{24}$ Another meta-analysis containing 4,243 patients from China, Singapore, South Korea, United Kingdom, and United States also showed that $17.6 \%$ had gastrointestinal symptoms, anorexia in $26.8 \%$, diarrhea in $12.5 \%$, nausea and vomiting in $10.2 \%$, and abdominal pain or discomfort in $9.2 \% .^{25}$ The frequencies of diarrhea have varied from $2.0 \%$ to $10.1 \%$ and nausea and/ or vomiting varied from $1.0 \%$ to $10.1 \%$ in different studies. ${ }^{8,26-28}$

\section{Fecal-oral transmission}

Wang et al. ${ }^{29}$ found infectious virions of SARS-CoV -2 in feces, and Amirian et al. ${ }^{30}$ detected the viral RNA in feces and sewage. During the 2003 outbreak, the Xiao Tang Shan Hospital and 309th Hospital of PLA first reported SARS-CoV RNA in wastewater. ${ }^{31}$ The first confirmed COVID-19 patient in the United States was on January 20, 2020, and had nausea and vomiting, with stool and respiratory specimens being positive for SARS-CoV-2 by real-time reverse transcriptase polymerase chain reaction. ${ }^{15}$ Hospitals in China also reported a number of COVID-19 patients positive for SARSCoV-2 in fecal virus tests. ${ }^{32-34} \mathrm{At}$ the same time, studies showed that COVID-19 patients could remain positive (via fecal virus test) after respiratory symptoms disappeared and negative throat swab test was obtained. ${ }^{14}$ The study of Ong et al. ${ }^{35}$ showed that samples from a COVID-19 patient with confirmed fecal positivity and no diarrhea, taken from the surface of the toilet bowl, inside bowl 
of the sink and the door handle, were positive for SARS-CoV-2, while post-cleaning samples were negative. Cheung et al. ${ }^{25}$ proved that viable virus existed for at least 3 hours in aerosols after their formation, and for up to 2 or 3 days on plastic and stainless steel surfaces. Based on the high viral infectivity of SARS-CoV- $2,{ }^{3}$ one study found that exposure to a fecal-polluted environment might cause "fecal-aerosol-respiratory transmission". ${ }^{32}$ All of these facts suggest that, apart from the classic respiratory droplets and close contact transmission of the virus, we should not ignore the "fecaloral" and "fecal-aerosol-respiratory" transmission routes. Preventing the spread of diseases, we should cut off such.

\section{Possible mechanisms of gastrointestinal injury}

ACE2 is the receptor used by SARS-CoV to enter host cells. Genome sequencing test indicated that SARS-CoV-2 shared $79.6 \%$ sequence identity to SARS-CoV; moreover, the sequences encode and express the spike glycoproteins which can combine with the entry receptor ACE2 to help these viruses enter human cells..$^{36,37}$ The spike protein is a ligand on the SARS-CoV-2 surface that can combine with ACE-2. Many research studies have provided evidence to support that ACE2 is the same cell entry receptor for the spike protein of SARS-CoV-2 and SARS-CoV, ${ }^{38}$ and its mRNA and protein are highly expressed in lung and the small intestinal enterocytes but not in the goblet cells or intestinal immune cells. ${ }^{39,40}$ Gut cells in contact with food and foreign pathogens directly highly express ACE2 on the surface in the digestive tract, which might lead to inflammation susceptibility and gastrointestinal symptoms. ACE2 expression on the surface cells of the small intestine might mediate the invasion of the virus and amplification the activation of gastrointestinal inflammation, which might be a possible mechanism of digestive symptoms in COVID-19 patients and explain the presence of the virus in patients' stool samples. ${ }^{40}$

A study showed that ACE2 played a significant role in amino acid homeostasis and in maintaining the intestinal microbiota. ${ }^{41}$ The ACE2 knockout mouse model showed a decline in the uptake of tryptophan, which resulted in decreasing the expression of antimicrobial peptides from small intestinal Paneth cells, changes in the intestinal microbiota, and ultimately in a high sensitivity to colitis, but all of those disturbances were restored by tryptophan supplementation. ${ }^{42}$ This might further explain the diarrhea observed in COVID-19 patients.

Another possible explanation for diarrhea in COVID-19 patients is an intestinal flora disturbance. A prospective, randomized, controlled study investigated changes in the fecal microbiota in COVID-19 patients. ${ }^{43}$ The authors compared microbiome data between $15 \mathrm{COV}$ ID-19 patients, 6 subjects with community-acquired pneumonia, and 15 healthy individuals (controls). At the time of hospitalization and at all timepoints during hospitalization, patients with COVID-19 had significant alterations in fecal microbiomes compared with controls, and characterized by enrichment of opportunistic pathogens and depletion of beneficial commensals. Depleted symbionts and gut dysbiosis persisted even after clearance of SARS-CoV-2 (throat swabnegative) and resolution of respiratory symptoms.

\section{Treatment and protection}

There has been no exact evidence on the efficacy of drugs in the therapy of gastrointestinal symptoms that caused by SARS-CoV-2. Reviewing relative studies, we have summarized here potential therapies, for which safety and efficiency need more clinical study to confirm.
Studies have suggested that the use of ACE2 inhibitors might reduce direct damage to the gastrointestinal tract caused by coronavirus. Hashimoto et al. ${ }^{41}$ reported that ACE2 inhibitors could reduce the inflammatory state of mouse intestinal tract, and ACE2 might be a key target for the treatment of COVID-19. In the diagnosis and treatment scheme of COVID-19, chloroquine was included in the therapeutic drug category. ${ }^{44}$ Similar to chloroquine, azathioprine was an immunosuppressant and could inhibit vaccinia virus in vitro. ${ }^{45}$ Screening by bioinformatics suggested that azathioprine might be a potential ACE2 inhibitor. ${ }^{46}$ Directly binding to ACE2 or the coronavirus' spike glycoproteins could directly prevent invasion of the virus. ${ }^{47}$ Clinical trials of recombinant human ACE2 inhibitors are currently underway and have shown efficacy in animal studies.

$\mathrm{ACE}$ inhibitor/angiotensin receptor blocker (ACEI/ARB) is an antagonist of the renin-angiotensin-aldosterone system. Compared with non-ACEI/ARB patients, patients who were in the ACEI/ ARB group had a significantly lower risk of gastrointestinal symptoms and abnormal liver function throughout the disease course. ${ }^{48}$ Zhang et al. ${ }^{49}$ reported that ACEI/ARB could upregulate ACE2 expression, which might increase the entry of SARS-CoV-2. Thus, more clinical data are needed to confirm the efficacy of ACEI/ARB in COVID-19 patients.

Direct viral injury or drug use could cause intestinal flora disorders, which could also result in gastrointestinal symptoms. Gut dysbiosis can interfere with distant disorders ${ }^{50}$ as well as with gut-lung axis; lung inflammation can also affect the gut microbiota. ${ }^{51}$ Zuo et al. ${ }^{43}$ demonstrated that Bacteroides species might have a potential protective role in combating SARS-CoV-2 infection by hampering host entry through ACE2 but that gut Erysipelotrichaceae might augment SARS-CoV-2 infection in the host gut. Therefore, probiotics might be another good option for the treatment of COVID-19.

Patients with inflammatory bowel disease and other autoimmune diseases are considered a high-risk group for COVID-19, for they are more likely to take immune-suppressive or immunemodulatory treatment. For these patients, the use of biologicals have been suggested to modulate the immune system, such as Baricitinib, a JAK inhibitor, which can reduce the viral passage into the host cell and reduce inflammatory. ${ }^{52}$

Zhou et al. ${ }^{36}$ reported that, in symptomatic and asymptomatic patients, nasal swabs yielded higher viral loads than throat swabs. Campione et al. ${ }^{53}$ reported that lactoferrin could reduce infection and inflammation, acting as the barrier of both respiratory and intestinal mucosa or reverting the iron disorders caused by the viral colonization, which could be used in asymptomatic or mildly symptomatic patients.

We believe that more attention should be paid to the gastrointestinal symptoms of COVID-19 patients. Ignoring the atypical clinical manifestations of COVID-19 patients might lead to more infection of people and exacerbate the epidemic. Patients with diarrhea were usually treated in the gastroenterology department. If the outpatient physicians were not sufficiently vigilant about the gastrointestinal symptoms of COVID-19 patients, especially before the onset of fever and respiratory symptoms, it will inevitably increase the infection rate among medical workers. On the other hand, gastroenterology endoscopy staff should pay more attention to such patients, for they are more likely to make physical contact with infected patients' gastric or intestinal fluids and feces during care, such as surgery. Therefore, clinicians and not only doctors working in fever and respiratory clinics should raise their awareness of protecting themselves, treat all suspected patients as confirmed cases, and take strict protective measures. Clinicians should wear standard protective clothing, investigate all patients' and their relatives' epidemiological history, collect their body fluid samples 
(nasopharyngeal swabs, feces, blood, etc.) for etiological testing by means that will minimize their own risk of infection, and wash hands or change gloves after each physician examination. After endoscopic surgery, all used endoscopes and the endoscopy room itself must undergo standardized disinfection. For patients who were cured and returned home, some of them could still test positive for SARS-CoV-2 RNA in feces, although their nucleic acid tests are negative by throat swab. We believe that feces might be a potential route to transmit SARS-CoV-2. Therefore, we recommended that patients discharged from the hospital who met the clinical cure standard should still pay strict attention to hand hygiene, disinfecting public toilets and sinks thoroughly, and avoiding sharing toilets with others. If permitting, we recommend fecal nucleic acid detection before discharge of any COVID-19 patient.

\section{Future directions}

COVID-19 is a common enemy to all mankind in the $21^{\text {st }}$ century. Further research studies are needed to reveal the specific mechanism of gastrointestinal injury and the effective and safe treatment measures. In order to effectively prevent and treat the disease, find the source of infection early, and cut off the possible transmission routes, it is important to pay attention to the gastrointestinal manifestations and fecal-oral transmission of SARS-CoV-2. Physicians, especially gastroenterologists, should be highly vigilant, make definite diagnosis as soon as possible, and take care of themselves. In terms of disease treatment, further clinical studies are needed to clarify the efficacy and safety of ACEI/ARB. It is necessary to further research other receptors by which viruses can invade host cells, in order to guide clinical targeted therapy. In addition, the intestinal flora is the second genome of the human body and participates in immune regulation. It is necessary to clarify the efficacy and safety of probiotic therapy for the treatment of COVID-19 patients.

\section{Conclusions}

Although we do not know the exact source of the virus in the digestive system yet, we believe that fecal-oral transmission is possible. In clinical work, we should pay attention to atypical gastrointestinal symptoms and treat these patients actively and effectively. We should also adapt to research ACE2 inhibitors and probiotics for the treatment of COVID-19. In addition, we recommend taking early measures to prevent the spread of COVID-19 in hospitals and communities.

\section{Acknowledgments}

None.

\section{Funding}

Natural Science Foundation of Gansu Province, China (18JR3RA366).

\section{Conflict of interest}

The authors declare that there are no conflicts of interest.
Author contributions

Study design (TH, LQL, YQW), manuscript writing (TH, LQL), critical revision (TH, LQL, WMS, YPW); critical funding (WMS, YPW); administration (YL, TBH, HYZ); technical or material support (YQW, ZPW, WMS, YPW).

\section{References}

[1] Gorbalenya AE, Baker SC, Baric RS, de Groot RJ, Drosten C, Gulyaeva $\mathrm{AA}$, et al. The species Severe acute respiratory syndrome-related coronavirus: classifying 2019-nCoV and naming it SARS-CoV-2. Nat Microbiol 2020;5:536-544. doi:10.1038/s41564-020-0695-z.

[2] Qu G, Li X, Hu L, Jiang G. An Imperative Need for Research on the Role of Environmental Factors in Transmission of Novel Coronavirus (COVID-19). Environ Sci Technol 2020;54(7):3730-3732. doi:10.1021/acs. est.0c01102.

[3] Sanche S, Lin YT, Xu C, Romero-Severson E, Hengartner N, Ke R. High Contagiousness and Rapid Spread of Severe Acute Respiratory Syndrome Coronavirus 2. Emerg Infect Dis 2020;26(7):1470-1477. doi:10.3201/eid2607.200282.

[4] Health Commission. Notice on Novel Coronavirus Pneumonia Diagnosis and Treatment Guideline (trial version 8) (in Chinese). Available from: http://www.gov.cn/zhengce/zhengceku/2020-08/19/content_5535757.htm. Assessed 18 August, 2020.

[5] Caussy C, Tripathi A, Humphrey G, Bassirian S, Singh S, Faulkner C, et al. A gut microbiome signature for cirrhosis due to nonalcoholic fatty liver disease. Nat Commun 2019;10(1):1406. doi:10.1038/s41467019-09455-9.

[6] Xu X, Chen P, Wang J, Feng J, Zhou H, Li X, et al. Evolution of the novel coronavirus from the ongoing Wuhan outbreak and modeling of its spike protein for risk of human transmission. Sci China Life Sci 2020;63(3):457-460. doi:10.1007/s11427-020-1637-5.

[7] Li MY, Li L, Zhang Y, Wang XS. Expression of the SARS-CoV-2 cell receptor gene ACE2 in a wide variety of human tissues. Infect Dis Poverty 2020;9(1):45. doi:10.1186/s40249-020-00662-x.

[8] Huang C, Wang Y, Li X, Ren L, Zhao J, Hu Y, et al. Clinical features of patients infected with 2019 novel coronavirus in Wuhan, China. Lancet 2020;395(10223):497-506. doi:10.1016/S0140-6736(20)30183-5.

[9] Aggarwal G, Cheruiyot I, Aggarwal S, Wong J, Lippi G, Lavie CJ, et al. Association of Cardiovascular Disease With Coronavirus Disease 2019 (COVID-19) Severity: A Meta-Analysis. Curr Probl Cardiol 2020;45(8):100617. doi:10.1016/j.cpcardiol.2020.100617.

[10] Khashkhusha TR, Chan JSK, Harky A. ACE inhibitors and COVID-19: We don't know yet. J Card Surg 2020;35(6):1172-1173. doi:10.1111/ jocs.14582

[11] Carnevale S, Beretta P, Morbini P. Direct endothelial damage and vasculitis due to SARS-CoV-2 in small bowel submucosa of COVID-19 patient with diarrhea. J Med Virol 2020. doi:10.1002/jmv.26119.

[12] Gu J, Han B, Wang J. COVID-19: Gastrointestinal Manifestations and Potential Fecal-Oral Transmission. Gastroenterology 2020;158(6):1518-1519. doi:10.1053/j.gastro.2020.02.054.

[13] Zhao D, Yao F, Wang L, Zheng L, Gao Y, Ye J, et al. A Comparative Study on the Clinical Features of Coronavirus 2019 (COVID-19) Pneumonia With Other Pneumonias. Clin Infect Dis 2020;71(15):756-761. doi:10.1093/cid/ciaa247.

[14] Xiao F, Tang M, Zheng X, Liu Y, Li X, Shan H. Evidence for Gastrointestinal Infection of SARS-CoV-2. Gastroenterology 2020;158(6):18311833.e3. doi:10.1053/j.gastro.2020.02.055.

[15] Holshue ML, DeBolt C, Lindquist S, Lofy KH, Wiesman J, Bruce H, et al. First Case of 2019 Novel Coronavirus in the United States. N Engl J Med 2020;382(10):929-936. doi:10.1056/NEJMoa2001191.

[16] Barrantes FJ. While We Wait for a Vaccine Against SARS-CoV-2, Why Not Think About Available Drugs? Front Physiol 2020;11:820. doi:10.3389/fphys.2020.00820.

[17] Wang D, Hu B, Hu C, Zhu F, Liu X, Zhang J, et al. Clinical Characteristics of 138 Hospitalized Patients With 2019 Novel Coronavirus-Infected Pneumonia in Wuhan, China. JAMA 2020;323(11):1061-1069. 
doi:10.1001/jama.2020.1585.

[18] Peiris JS, Chu CM, Cheng VC, Chan KS, Hung IF, Poon LL, et al. Clinical progression and viral load in a community outbreak of coronavirus-associated SARS pneumonia: a prospective study. Lancet 2003;361(9371):1767-1772. doi:10.1016/s0140-6736(03)13412-5.

[19] Bossi MM, Tufoni M, Zaccherini G, Antognoli A, Domenicali M, Caraceni P. A web-based group treatment for patients with alcoholic liver diseases at the time of the COVID-19 pandemic. Dig Liver Dis 2020;52(9):956-957. doi:10.1016/j.dld.2020.04.023.

[20] Zhang JJ, Dong X, Cao YY, Yuan YD, Yang YB, Yan YQ, et al. Clinical characteristics of 140 patients infected with SARS-CoV-2 in Wuhan, China. Allergy 2020;75(7):1730-1741. doi:10.1111/all.14238.

[21] Jin X, Lian JS, Hu JH, Gao J, Zheng L, Zhang YM, et al. Epidemiological, clinical and virological characteristics of 74 cases of coronavirus-infected disease 2019 (COVID-19) with gastrointestinal symptoms. Gut 2020;69(6):1002-1009. doi:10.1136/gutjnl-2020-320926.

[22] Li LQ, Huang T, Wang YQ, Wang ZP, Liang Y, Huang TB, et al. COVID-19 patients' clinical characteristics, discharge rate, and fatality rate of meta-analysis. J Med Virol 2020;92(6):577-583. doi:10.1002/ jmv. 25757.

[23] Guan WJ, Ni ZY, Hu Y, Liang WH, Ou CQ, He JX, et al. Clinical Characteristics of Coronavirus Disease 2019 in China. N Engl J Med 2020;382(18):1708-1720. doi:10.1056/NEJMoa2002032.

[24] Zarifian A, Zamiri Bidary M, Arekhi S, Rafiee M, Gholamalizadeh H, Amiriani A, et al. Gastrointestinal and hepatic abnormalities in patients with confirmed COVID-19: A systematic review and meta-analysis. J Med Virol 2020. doi:10.1002/jmv.26314.

[25] Cheung KS, Hung IFN, Chan PPY, Lung KC, Tso E, Liu R, et al. Gastrointestinal Manifestations of SARS-CoV-2 Infection and Virus Load in Fecal Samples From a Hong Kong Cohort: Systematic Review and Meta-analysis. Gastroenterology 2020;159(1):81-95. doi:10.1053/j. gastro.2020.03.065.

[26] Chen N, Zhou M, Dong X, Qu J, Gong F, Han Y, et al. Epidemiological and clinical characteristics of 99 cases of 2019 novel coronavirus pneumonia in Wuhan, China: a descriptive study. Lancet 2020;395(10223):507-513. doi:10.1016/s0140-6736(20)30211-7.

[27] Liu K, Fang YY, Deng Y, Liu W, Wang MF, Ma JP, et al. Clinical characteristics of novel coronavirus cases in tertiary hospitals in Hubei Province. Chin Med J (Engl) 2020;133(9):1025-1031. doi:10.1097/ CM9.0000000000000744.

[28] Zhou F, Yu T, Du R, Fan G, Liu Y, Liu Z, et al. Clinical course and risk factors for mortality of adult inpatients with COVID-19 in Wuhan, China: a retrospective cohort study. Lancet 2020;395(10229):1054-1062. doi:10.1016/S0140-6736(20)30566-3.

[29] Wang W, Xu Y, Gao R, Lu R, Han K, Wu G, et al. Detection of SARS-CoV-2 in Different Types of Clinical Specimens. JAMA 2020;323(18):18431844. doi:10.1001/jama.2020.3786.

[30] Amirian ES. Potential fecal transmission of SARS-CoV-2: Current evidence and implications for public health. Int J Infect Dis 2020;95:363370. doi:10.1016/j.ijid.2020.04.057.

[31] Wang XW, Li J, Guo T, Zhen B, Kong Q, Yi B, et al. Concentration and detection of SARS coronavirus in sewage from Xiao Tang Shan Hospital and the 309th Hospital of the Chinese People's Liberation Army. Water Sci Technol 2005;52(8):213-221. doi:10.2166/wst.2005.0266.

[32] Zhang W, Du RH, Li B, Zheng XS, Yang XL, Hu B, et al. Molecular and serological investigation of 2019-nCoV infected patients: implication of multiple shedding routes. Emerg Microbes Infect 2020;9(1):386389. doi:10.1080/22221751.2020.1729071.

[33] Xu Y, Li X, Zhu B, Liang H, Fang C, Gong Y, et al. Characteristics of pediatric SARS-CoV-2 infection and potential evidence for persistent fecal viral shedding. Nat Med 2020;26(4):502-505. doi:10.1038/s41591020-0817-4.

[34] Tang A, Tong ZD, Wang HL, Dai YX, Li KF, Liu JN, et al. Detection of Novel Coronavirus by RT-PCR in Stool Specimen from Asymptomatic Child, China. Emerg Infect Dis 2020;26(6):1337-1339. doi:10.3201/ eid2606.200301.

[35] Ong SWX, Tan YK, Chia PY, Lee TH, Ng OT, Wong MSY, et al. Air, Surface Environmental, and Personal Protective Equipment Contamination by Severe Acute Respiratory Syndrome Coronavirus 2 (SARS-CoV-2) From a Symptomatic Patient. JAMA 2020;323(16):1610-1612. doi:10.1001/jama.2020.3227.
[36] Zhou P, Yang XL, Wang XG, Hu B, Zhang L, Zhang W, et al. A pneumonia outbreak associated with a new coronavirus of probable bat origin. Nature 2020;579(7798):270-273. doi:10.1038/s41586-0202012-7.

[37] Lu R, Zhao X, Li J, Niu P, Yang B, Wu H, et al. Genomic characterisation and epidemiology of 2019 novel coronavirus: implications for virus origins and receptor binding. Lancet 2020;395(10224):565-574. doi:10.1016/s0140-6736(20)30251-8.

[38] Wu F, Zhao S, Yu B, Chen YM, Wang W, Song ZG, et al. A new coronavirus associated with human respiratory disease in China. Nature 2020;579(7798):265-269. doi:10.1038/s41586-020-2008-3.

[39] Wan Y, Shang J, Graham R, Baric RS, Li F. Receptor Recognition by the Novel Coronavirus from Wuhan: an Analysis Based on Decade-Long Structural Studies of SARS Coronavirus. J Virol 2020;94(7):e0012720. doi:10.1128/jvi.00127-20.

[40] Zhang H, Li HB, Lyu JR, Lei XM, Li W, Wu G, et al. Specific ACE2 expression in small intestinal enterocytes may cause gastrointestinal symptoms and injury after 2019-nCoV infection. Int J Infect Dis 2020;96:19-24. doi:10.1016/j.ijid.2020.04.027.

[41] Hashimoto T, Perlot $T$, Rehman A, Trichereau J, Ishiguro H, Paolino $\mathrm{M}$, et al. ACE2 links amino acid malnutrition to microbial ecology and intestinal inflammation. Nature 2012;487(7408):477-481. doi:10.1038/nature11228.

[42] He F, Wu C, Li P, Li N, Zhang D, Zhu Q, et al. Functions and Signaling Pathways of Amino Acids in Intestinal Inflammation. BioMed Research Int 2018;2018:9171905. doi:10.1155/2018/9171905.

[43] Zuo T, Zhang F, Lui GCY, Yeoh YK, Li AYL, Zhan H, et al. Alterations in Gut Microbiota of Patients With COVID-19 During Time of Hospitalization. Gastroenterology 2020;159(3):944-955.e8. doi:10.1053/j. gastro.2020.05.048.

[44] Medical administration and Hospital Authority. Notice on Novel Coronavirus Pneumonia Diagnosis and Treatment Guideline (trial version 7) (in Chinese). Available from: http://www.nhc.gov.cn/yzygj/s7653p/2 02003/46c9294a7dfe4cef80dc7f5912eb1989.shtml. Assessed 3 May, 2020.

[45] Damaso CR, Oliveira MF, Massarani SM, Moussatché N. Azathioprine inhibits vaccinia virus replication in both BSC-40 and RAG cell lines acting on different stages of virus cycle. Virology 2002;300(1):79-91. doi:10.1006/viro.2002.1534.

[46] Chen Y, Guo Y, Pan Y, Zhao ZJ. Structure analysis of the receptor binding of 2019-nCoV. Biochem Biophys Res Commun 2020;525(1):135140. doi:10.1016/j.bbrc.2020.02.071

[47] Zhang $\mathrm{H}$, Penninger JM, Li $\mathrm{Y}$, Zhong $\mathrm{N}$, Slutsky AS. Angiotensinconverting enzyme 2 (ACE2) as a SARS-CoV-2 receptor: molecular mechanisms and potential therapeutic target. Intensive Care Med 2020;46(4):586-590. doi:10.1007/s00134-020-05985-9.

[48] Tan ND, Qiu Y, Xing XB, Ghosh S, Chen MH, Mao R. Associations Between Angiotensin-Converting Enzyme Inhibitors and Angiotensin II Receptor Blocker Use, Gastrointestinal Symptoms, and Mortality Among Patients With COVID-19. Gastroenterology 2020;159(3):1170-1172.e1. doi:10.1053/j.gastro.2020.05.034.

[49] Zhang P, Zhu L, Cai J, Lei F, Qin JJ, Xie J, et al. Association of Inpatient Use of Angiotensin-Converting Enzyme Inhibitors and Angiotensin II Receptor Blockers With Mortality Among Patients With Hypertension Hospitalized With COVID-19. Circ Res 2020;126(12):1671-1681. doi:10.1161/CIRCRESAHA.120.317134.

[50] Avery ME, Snyder JD. Oral therapy for acute diarrhea. The underused simple solution. N Engl J Med 1990;323(13):891-894. doi:10.1056/ NEJM199009273231307.

[51] Dumas A, Bernard L, Poquet Y, Lugo-Villarino G, Neyrolles O. The role of the lung microbiota and the gut-lung axis in respiratory infectious diseases. Cell Microbiol 2018;20(12):e12966. doi:10.1111/ cmi.12966.

[52] Richardson P, Griffin I, Tucker C, Smith D, Oechsle O, Phelan A, et al. Baricitinib as potential treatment for 2019-nCoV acute respiratory disease. Lancet 2020;395(10223):e30-e31. doi:10.1016/S01406736(20)30304-4.

[53] Campione E, Cosio T, Rosa L, Lanna C, Di Girolamo S, Gaziano R, et al. Lactoferrin as Protective Natural Barrier of Respiratory and Intestinal Mucosa against Coronavirus Infection and Inflammation. Int J Mol Sci 2020;21(14):4903. doi:10.3390/ijms21144903. 